\title{
Robust Path Following Control for Small Electric Vehicles Equipped with In-Wheel Motors
}

\author{
- Application to the JSAE-SICE Benchmark Problem No. 3 -
}

\author{
Takatsugu Oda $^{\text {1) }}$ Kenichiro Nonaka $^{2)}$ Kazuma Sekiguchi $^{2)}$ \\ 1) Graduate School of Engineering, Tokyo City University, \\ 1-28-1 Tamazutsumi, Setagaya, Tokyo, 158-8557, Japan (E-mail: taka1107@gmail.com) \\ 2) Faculty of Engineering, Tokyo City University, \\ 1-28-1 Tamazutsumi, Setagaya,Tokyo, 158-8557, Japan(E-mail: knonaka@tcu.ac.jp, ksekiguc@tcu.ac.jp)
}

Received on November 11, 2016

\begin{abstract}
In this paper, it is demonstrated that the proposed robust path following controller enhances maneuverability and stability of small electric vehicles (EVs) equipped with in-wheel motors through the JSAE-SICE benchmark problem No. 3. In order to achieve high maneuverability, maximum tire force is explicitly considered using octagonal approximation. Verifications are conducted using full vehicle model so that the practical vehicle performance is evaluated. In addition to evaluate the total vehicle performance, we apply the controller to all benchmark scenarios and show its maneuverability and stability of small EV are enhanced.
\end{abstract}

KEY WORDS: Vehicle dynamics, Electronic stability control / JSAE-SICE benchmark problem, Autonomous control [B1]

\section{Introduction}

Small electric vehicles $(\mathrm{EV})$ have attracted a lot of interest as a powerful solution to energy and environmental problems because the vehicles do not emit pollutants. In addition, low rolling resistance coefficient $(\mathrm{RRC})$ tires that improve vehicle fuel efficiency also have attracted many attentions. Small EVs equipped with low RRC tires, however, tend to deteriorate its maneuverability and stability because a small vehicle has small tire force limitation and small inertia, which tend to sensitive to disturbance, while low RRC tires have weak tire lateral stiffness (1). These problems are dealt with as a benchmark problem given by the vehicle modeling and control committee of the society of automotive engineers of japan (JSAE) and the society of instrument and control engineers (SICE) ${ }^{(2)}$. The benchmark problem focuses on the new generation four wheels driving and steering (4WDS) vehicles as a controlled vehicle and requests to achieve the same maneuverability and stability of those small EV equipped with low RRC tires as conventional vehicles by designing an advanced control system. Since cooperation of redundant input has possibility to enhance maneuverability ${ }^{(3)}$, 4WDS vehicles have attracted many interests. Mokhiamar et al. proposed the optimum coordination of 4WDS vehicle based on tire workload ${ }^{(4)}$. Analytical results for maneuverability of 4WDS vehicle are shown by Ono et al. ${ }^{(5)}$.

In practical vehicles, vehicle maneuverability is limited by physical constraints mainly due to tire force. Hence model predictive control (MPC) that can explicitly deals with constraints is an efficient choice. Falcone et al. considered constraints of torques and steering angles ${ }^{(6)}$. Beal et al. proposed a controller that constrains vehicle states to keep it in a stable region ${ }^{(7)}$. Attia et al. proposed a nonlinear MPC considering complex system characteristic including power-train dynamics ${ }^{(8)}$.

On the other hand, it is important to deal with effects of disturbance and model uncertainty. It is why many robust vehicle control techniques are studied. For example, Canale et al. proposed higher order sliding mode controller (SMC) considering chattering avoidance ${ }^{(9)}$. He et al. proposed a $H_{\infty}$ control method to attenuate the effect of external disturbance ${ }^{(10)}$.

In order to enhance vehicle maneuverability and stability, Oda et al. proposed a robust path following controller ${ }^{(11)}$. In this method MPC is introduced, because considering the friction circle is important to achieve high maneuverability. On the other hands, since MPC is the predictive method based on mathematical model, the effect of unexpected disturbance and model uncertainty might be severe. Then, SMC is also introduced to suppress the undesired effect. Although the verification in the previous study ${ }^{(11)}$ is conducted using only planar vehicle model, in practical condition, vehicles are affected by un-modeled factor like running resistance and suspension. Hence in this paper, we evaluate the practical performance by using a detailed vehicle model.

As tackling the JSAE-SICE benchmark problem that deals with new generation EVs equipped with in-wheel motors (IWMs), some researcher applied their controllers. Yamaguchi et al. applied a hierarchical controller based on model matching method (12). Yun et al. analyzed the frequency response of the new generation EVs and applied a gain scheduled $H_{\infty}$ controller ${ }^{(13)}$. No one, however, has successfully applied controller to all 
benchmark scenarios using full vehicle model yet. In this paper, we apply the proposed controller to all benchmark scenarios using full vehicle model and show the maneuverability and stability of new generation EVs are enhanced.

The main contributions of this paper are as follows: (i) the octagonal approximation of complex friction circle is proposed in order to consider the tire force limitation, (ii) the robust path following controller ${ }^{(11)}$ is extended to traction and camber control in order to bring out the performance of the new generation EV, and (iii) It is shown a first result successfully applied to all test scenarios of the JSAE-SICE benchmark problem No. 3 using full vehicle model. This paper is organized as follows: Section 2 presents the vehicle and tire models. The proposed robust path tracking controller is described in Section 3. The detail of JSAESICE benchmark problem and the simulation results are described in Section 4. Finally, the conclusions are presented.

\section{Vehicle model}

\subsection{Vehicle model}

Figure 1 depicts 4WDS vehicle model. The vehicle dynamics is given as follows:

$$
\begin{gathered}
\frac{\mathrm{d}}{\mathrm{d} t} x=v \cos (\theta+\beta), \\
\frac{\mathrm{d}}{\mathrm{d} t} y=v \sin (\theta+\beta), \\
\frac{\mathrm{d}}{\mathrm{d} t} \theta=\gamma, \\
m \frac{\mathrm{d}}{\mathrm{d} t} v=F_{v}+d_{v}, \\
m v \frac{\mathrm{d}}{\mathrm{d} t}(\theta+\beta)=F_{\beta}+d_{\beta}, \\
I_{z} \frac{\mathrm{d}}{\mathrm{d} t} \gamma=F_{\gamma}+d_{\gamma},
\end{gathered}
$$

where $m$ denotes total mass of the vehicle, $I_{z}$ is inertia moment about the vertical axis, $(x, y)$ are position of $\mathrm{CoG}, \theta$ is attitude angle, $\gamma$ is yaw rate and $\beta$ is side slip angle, respectively. $d_{v}, d_{\beta}$ and $d_{\gamma}$ are unknown disturbances, representing wind disturbance and model uncertainties. $F_{v}, F_{\beta}$ and $F_{\gamma}$ are longitudinal, lateral and rotational generalized force defined as follows:

$$
\begin{gathered}
F_{v}=\sum_{i=1}^{4}\left(\hat{f}_{x i} \cos \beta+\hat{f}_{y i} \sin \beta\right), \\
F_{\beta}=\sum_{i=1}^{4}\left(-\hat{f}_{x i} \sin \beta+\hat{f}_{y i} \cos \beta\right), \\
F_{\gamma}=\sum_{i=1}^{4}\left(w_{i} \hat{f}_{x i}+l_{i} \hat{f}_{y i}\right),
\end{gathered}
$$

where $\hat{f}_{x i}$ and $\hat{f}_{y i}$ are forces generated by $i$-th tires. $i$ is $1, \cdots, 4$ corresponding to front-left, front-right, rear-left, and rear-right wheel, respectively. $\left(l_{i}, w_{i}\right)$ are the position of each tire in the body fixed coordinate. Using the distance between front or rear wheel and CoG $l_{f}, l_{r}$ and the half length of tread $w_{i}, l_{1}=l_{2}=l_{f}$, $l_{3}=l_{4}=-l_{r}, w_{1}=w_{3}=w, w_{2}=w_{4}=-w$ is established. Tire forces of attitude angle direction component $\hat{f}_{x i}$ and $\hat{f}_{y i}$ are calculated based on a tire model that expresses the tire force of longitudinal and lateral direction $f_{x i}$ and $f_{y i}$ and the steering angle $\delta_{i}$ as follows:

$$
\begin{aligned}
& \hat{f}_{x i}=f_{x i} \cos \delta_{i}-f_{y i} \sin \delta_{i}, \\
& \hat{f}_{y i}=f_{x i} \sin \delta_{i}+f_{y i} \cos \delta_{i} .
\end{aligned}
$$

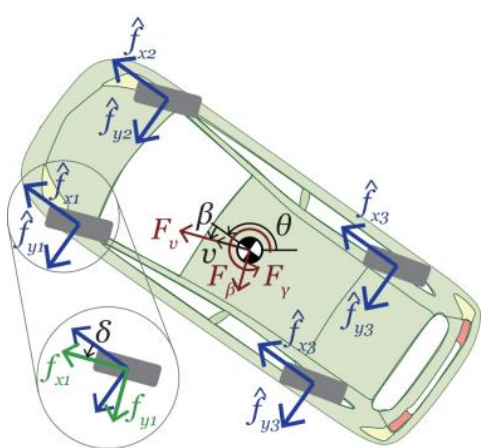

Fig. 1 Vehicle model

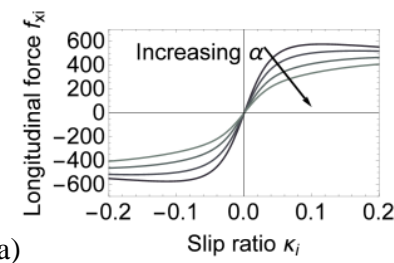

(a)

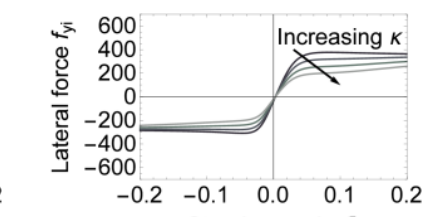

(b)
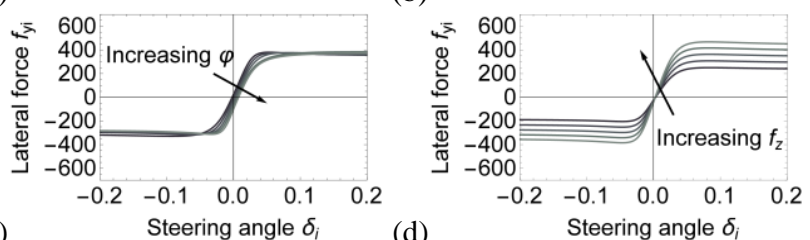

(d)

Fig. 2 Tire combined slip characteristics: (a) Longitudinal force at various tire slip angle $\alpha$. (b) Lateral force at various slip ratio $\kappa$. (c) Lateral force at various tire camber angle $\varphi$. (d) Lateral force at various vertical load $f_{z i}$.

\subsection{Tire model}

2.2.1 Magic formula ${ }^{(14)}$

In this section, we explain a nonlinear tire model. The definition of tire slip angle $\alpha_{i}$ is as follows:

$$
\alpha_{i}\left(\delta_{i}\right)=\tan ^{-1}\left(\frac{v_{y, i}}{v_{x, i}}\right)-\delta_{i},
$$

where $v_{x, i}$ and $v_{y, i}$ are wheel velocities. To consider nonlinearity of tire force $f_{x i}$ and $f_{y i}$, a Pacejka model is introduced. This semiempirical nonlinear mathematical model called magic formula is expressed as functions of slip ratio $\kappa_{i}$, tire slip angle $\alpha_{i}$, camber angle $\varphi_{i}$, vertical load $f_{z i}$ and friction coefficient $\mu_{i}$ of each tire. The tire model is expressed as follows:

$$
\begin{gathered}
f_{x i}\left(\kappa_{i}, \alpha_{i}, \varphi_{i}, f_{z i}, \mu_{i}\right)=f_{x 0}\left(\kappa_{i}, \varphi_{i}, f_{z i}, \mu_{i}\right) G_{x \alpha}, \\
f_{x 0}=D_{x} \sin \left(C _ { x } \operatorname { t a n } ^ { - 1 } \left(B_{x} \kappa_{x i}\right.\right. \\
\left.-E_{x}\left(B_{x} \kappa_{x i}-\tan ^{-1}\left(B_{x} \kappa_{x i}\right)\right)\right)+S_{V x}, \\
f_{y i}\left(\kappa_{i}, \alpha_{i}, \varphi_{i}, f_{z i}, \mu_{i}\right)=f_{y 0}\left(\alpha_{i}, \varphi_{i}, f_{z i}, \mu_{i}\right) G_{y \kappa}, \\
f_{y 0}=D_{y} \sin \left(C _ { y } \operatorname { t a n } ^ { - 1 } \left(B_{y} \alpha_{y i}\right.\right. \\
\left.\left.-E_{y}\left(B_{y} \alpha_{y i}-\tan ^{-1}\left(B_{y} \alpha_{y i}\right)\right)\right)\right)+S_{V y},
\end{gathered}
$$

where $f_{x 0}$ and $f_{y 0}$ are pure longitudinal/lateral slip model for either at side slip equal to zero $\left(\alpha_{i}=0\right)$ and free rolling $\left(\kappa_{i}=0\right)$. $G_{x \alpha}$ and $G_{y \kappa}$ are weighting nonlinear functions introduced to produce the interaction effects of $\alpha_{i}$ on $f_{x i}$ and of $\kappa_{i}$ on $f_{y i} . B_{j}, C_{j}$, $D_{j}, E_{j}, S_{V j}(j=x, y)$ are intermediate parameters, which express the tire characteristics. (See the literature ${ }^{(14)}$ for details.) Figure 2 (a) and (b) depict combined slip characteristics and indicate drop in the slope and peak. This phenomenon is well known as a concept of friction circles, which is kept even if camber angle $\varphi_{i}$ is added, as indicated in Fig. 2 (c). In addition, the size of friction circle depends on vertical load $f_{z i}$, as indicated in Fig. 2(d). Therefore it is important to consider the friction circle in order to generate large tire force. 


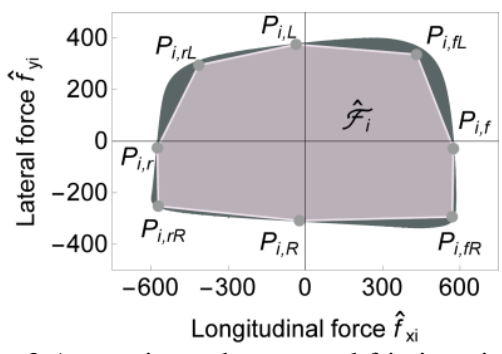

Fig. 3 Approximated octagonal friction circle

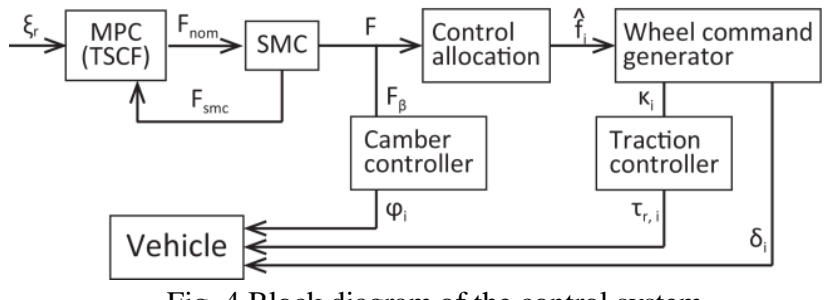

Fig. 4 Block diagram of the control system

\subsubsection{Approximation of the friction circle}

Considering friction circles is important, because the vehicle maneuverability is constrained by a maximum tire force. Since an outline of friction circle is hard to obtain due to the nonlinearity of the tire model, we introduce the octagonal approximation consisting of linear inequalities. In Fig. 3, the original and approximated friction circle and eight vertices $P_{i}$ of an octagonal approximated friction circle $\hat{\mathcal{F}}_{i}$ are described. Table 1 shows the $\hat{f}_{x i}$ and $\hat{f}_{y i}$ coordinate of each vertices $P_{i}$, where $\kappa_{i, f}, \kappa_{i, r}, \delta_{i, L}$ and $\delta_{i, R}$ are parameters calculated as follows:

$$
\begin{aligned}
\kappa_{i, f} & =\underset{\kappa}{\operatorname{argmax}} \hat{f}_{x i}\left(\kappa, \alpha_{i}(0), \varphi_{i}, f_{z i}, \mu_{i}\right), \\
\kappa_{i, r} & =\underset{\kappa}{\operatorname{argmin}} \hat{f}_{x i}\left(\kappa, \alpha_{i}(0), \varphi_{i}, f_{z i}, \mu_{i}\right), \\
\delta_{i, L} & =\underset{\delta}{\operatorname{argmax}} \hat{f}_{y i}\left(0, \alpha_{i}(\delta), \varphi_{i}, f_{z i}, \mu_{i}\right), \\
\delta_{i, R} & =\underset{\delta}{\operatorname{argmin}} \hat{f}_{y i}\left(0, \alpha_{i}(\delta), \varphi_{i}, f_{z i}, \mu_{i}\right) .
\end{aligned}
$$

It is noted that the coordinates of longitudinal and lateral force are rotated from wheel direction $f_{x i}$ and $f_{y i}$ to attitude angle direction $\hat{f}_{x i}$ and $\hat{f}_{y i}$, to express in body-fixed coordinate. Thus we obtain the octagonal friction circle by calculating only four optimization problems with one decision variable for one wheel.

\section{Control law design}

In this paper, we design the vehicle dynamics control law based on the robust path tracking control ${ }^{(11)}$ in which maximum tire forces are explicitly considered using MPC, while effect of disturbance is suppressed using SMC. The previous controller is extended on following points: considering approximated friction circle and introducing the traction and camber controller.

\subsection{Structure of the robust path tracking controller}

Figure 4 depicts the block diagram of the proposed control system. Details of each block are explained in the follow sections. It is noted that the approximated friction circles are calculated based on the current vertical load $f_{z i}$ and camber angle $\phi_{i}$ in order to consider the current friction circle.

\subsection{Time-state control form ${ }^{(11)}$}

In this section, we explain the time-state control form (TSCF) used in MPC block in Fig. 4. We introduce the path following control ${ }^{(11)}$ so as to evaluate the deviation from the target path.

The travel distance $s_{q}$ and the path following error $z$, which expresses the distance between vehicle position and target path, are defined as follows:
Table 1 Vertices of approximated octagonal friction circle

\begin{tabular}{ccc}
\hline Vertex & $\hat{\boldsymbol{f}}_{\boldsymbol{x}}$ - component & $\hat{\boldsymbol{f}}_{\boldsymbol{y}^{-} \text {component }}$ \\
\hline \hline$P_{i, f}$ & $\hat{f}_{x i}\left(\kappa_{i, f}, \alpha_{i}(0)\right)$ & $\hat{f}_{y i}\left(\kappa_{i, f}, \alpha_{i}(0)\right)$ \\
$P_{i, f L}$ & $\hat{f}_{x i}\left(\kappa_{i, f}, \alpha_{i}\left(\delta_{i, L}\right)\right)$ & $\hat{f}_{y i}\left(\kappa_{i, f}, \alpha_{i}\left(\delta_{i, L}\right)\right)$ \\
$P_{i, L}$ & $\hat{f}_{x i}\left(0, \alpha_{i}\left(\delta_{i, L}\right)\right)$ & $\hat{f}_{y i}\left(0, \alpha_{i}\left(\delta_{i, L}\right)\right)$ \\
$P_{i, r L}$ & $\hat{f}_{x i}\left(\kappa_{i, r}, \alpha_{i}\left(\delta_{i, L}\right)\right)$ & $\hat{f}_{y i}\left(\kappa_{i, r}, \alpha_{i}\left(\delta_{i, L}\right)\right)$ \\
$P_{i, r}$ & $\hat{f}_{x i}\left(\kappa_{i, r}, \alpha_{i}(0)\right)$ & $\hat{f}_{y i}\left(\kappa_{i, r}, \alpha_{i}(0)\right)$ \\
$P_{i, r R}$ & $\hat{f}_{x i}\left(\kappa_{i, r}, \alpha_{i}\left(\delta_{i, R}\right)\right)$ & $\hat{f}_{y i}\left(\kappa_{i, r}, \alpha_{i}\left(\delta_{i, R}\right)\right)$ \\
$P_{i, R}$ & $\hat{f}_{x i}\left(0, \alpha_{i}\left(\delta_{i, R}\right)\right)$ & $\hat{f}_{y i}\left(0, \alpha_{i}\left(\delta_{i, R}\right)\right)$ \\
$P_{i, f R}$ & $\hat{f}_{x i}\left(\kappa_{i, f}, \alpha_{i}\left(\delta_{i, R}\right)\right)$ & $\hat{f}_{y i}\left(\kappa_{i, f}, \alpha_{i}\left(\delta_{i, R}\right)\right)$ \\
\hline
\end{tabular}

$$
\begin{gathered}
\frac{\mathrm{d} s_{q}}{\mathrm{~d} t}=v, \\
\frac{\mathrm{d} z}{\mathrm{~d} t}=-v \sin (\tilde{\theta}+\tilde{\beta}),
\end{gathered}
$$

where $\tilde{\theta}+\tilde{\beta}:=(\theta+\beta)-\left(\theta_{r}+\beta_{r}\right)$ is tracking error of moving direction. We consider differential equation of vehicle velocity $v$, path following error $z$ and attitude angle $\theta$, with respect to travel distance $s_{q}$ as follows:

$$
\begin{gathered}
\frac{\mathrm{d} v}{\mathrm{~d} s_{q}}=\frac{\mathrm{dv}}{\mathrm{dt}} \frac{\mathrm{d} t}{\mathrm{~d} s_{q}}=: u_{v}, \\
\frac{\mathrm{d}^{2} z}{\mathrm{~d} s_{q}^{2}}=\frac{\mathrm{d}}{\mathrm{d} s_{q}}\left(\frac{\mathrm{d} z}{\mathrm{~d} t} \frac{\mathrm{d} t}{\mathrm{~d} s_{q}}\right)=: u_{z}, \\
\frac{\mathrm{d}^{2} \theta}{\mathrm{d} s_{q}^{2}}=\frac{\mathrm{d}}{\mathrm{d} s_{q}}\left(\frac{\mathrm{d} \theta}{\mathrm{d} t} \frac{\mathrm{d} t}{\mathrm{~d} s_{q}}\right)=: u_{\theta},
\end{gathered}
$$

where $u=\left[u_{v}, u_{z}, u_{\theta}\right]^{T}$ is virtual inputs for the path following control. The linearized dynamics Eqs. (21)(22)(23) are considered in MPC. In addition to consider the nominal vehicle model, let us temporarily suppose that $d_{v} \equiv 0, d_{\beta} \equiv 0$ and $d_{\gamma} \equiv 0$ in Eqs. (4)(5)(6). Using the above assumption, the relationship between nominal generalized forces $F_{\text {nom }}=\left[F_{v, \text { nom }}, F_{\beta, \text { nom }}, F_{\gamma, \text { nom }}\right]^{T}$ and three virtual inputs $u_{v}, u_{z}, u_{\theta}$ are described as follows:

$$
\begin{gathered}
F_{v, \text { nom }}=m v u_{v}, \\
F_{\beta, \text { nom }}=m v g_{\beta}, \\
F_{\gamma, \text { nom }}=I_{z} g_{\gamma},
\end{gathered}
$$

where

$$
\begin{gathered}
g_{\beta}=v\left(\frac{\kappa_{r}}{1+z \kappa_{r}} \cos (\tilde{\theta}+\tilde{\beta})-\frac{1}{\cos (\tilde{\theta}+\tilde{\beta})} u_{z}\right), \\
g_{\gamma}=v^{2} u_{\theta}+\gamma u_{v} .
\end{gathered}
$$

3.3 Model predictive control

To consider tire force limitations and to optimize the vehicle future behavior, we apply MPC, which is a kind of predictive control dealing with a constrained optimization problem.

Performance index is as follows:

$$
\begin{aligned}
J_{m p c} & =\sum_{i=1}^{T} \tilde{\xi}(k+i \mid k)^{T} Q(i) \tilde{\xi}(k+i \mid k) \\
& +\sum_{i=1}^{T-1} \Delta u(k+i \mid k)^{T} R(i) \Delta u(k+i \mid k),
\end{aligned}
$$

where $\xi:=\left[v, z, \mathrm{~d} z / \mathrm{d} s_{q}, \theta, \mathrm{d} \theta / \mathrm{d} s_{q}\right]^{T}$ is state, $\xi_{r}$ is its reference state, $\tilde{\xi}=\xi-\xi_{r}$ is state tracking error and $u:=\left[u_{v}, u_{z}, u_{\theta}\right]^{T}$ is virtual input. $Q(i)>0$ and $R(i) \geq 0$ are weighting matrices.

To consider the tire force limitation, we introduce constraints for the virtual inputs as follows:

$$
\left[\begin{array}{l}
F_{v, \text { nom }}+F_{v, s m c} \\
F_{\beta, \text { nom }}+F_{\beta, s m c} \\
F_{\gamma, \text { nom }}+F_{\gamma, s m c}
\end{array}\right]=:\left[\begin{array}{l}
F_{v} \\
F_{\beta} \\
F_{\gamma}
\end{array}\right] \in \mathcal{U},
$$

where $F_{s m c}=\left[F_{v, s m c}, F_{\beta, s m c}, F_{\gamma, s m c}\right]^{T}$ is the input generated in $\mathrm{SMC}$, and the detail is described in section 3.4. The feasible generalized force set $\mathcal{U}$ is expressed as a hexadecahedron 
considering ten vertices on $\left(F_{v}, F_{\beta}, F_{\gamma}\right)$ space, as indicated in Fig. 5. To calculate the set $\mathcal{U}$, we introduce the eight vertices depicted in Fig. 5 (a). The vertices that have the following component are calculated based on approximated friction circle $\widehat{\mathcal{F}}_{i}$ :

$$
P_{j}^{k}=\sum_{i=1}^{4} P_{i, j}^{k} \quad(j \in\{f, f L, L, r L, r, r R, R, f R\}),
$$

where superscript $k \in\left\{F_{v}, F_{\beta}\right\}$ is the $F_{v}$ and $F_{\beta}$ component of the vertices $P_{j}$, respectively. It is noted that the $F_{\gamma}$ components of these vertices are determined by the $F_{v}$ and $F_{\beta}$ components. Then the $F_{\gamma}$ component of vertices $P_{T}$ and $P_{B}$ are computed from the following equations:

$$
\begin{array}{r}
P_{T}^{F_{\gamma}}=F_{\gamma}\left(\kappa_{1, r}, \alpha_{1}\left(\delta_{1, L}\right), \kappa_{2, f}, \alpha_{2}\left(\delta_{2, L}\right),\right. \\
\left.\kappa_{3, r}, \alpha_{3}\left(\delta_{3, R}\right), \kappa_{4, f}, \alpha_{4}\left(\delta_{4, R}\right)\right), \\
P_{B}^{F_{\gamma}}=F_{\gamma}\left(\kappa_{1, f}, \alpha_{1}\left(\delta_{1, R}\right), \kappa_{2, r}, \alpha_{2}\left(\delta_{2, R}\right),\right. \\
\left.\kappa_{3, f}, \alpha_{3}\left(\delta_{3, L}\right), \kappa_{4, r}, \alpha_{4}\left(\delta_{4, L}\right)\right) .
\end{array}
$$

It is noted that $F_{v}$ and $F_{\beta}$ components of the vertices $P_{T}$ and $P_{B}$ are also uniquely defined. Thus using these vertices, the feasible generalized force set $\mathcal{U}$ is obtained, as indicated in Fig. 5 (b).

In summary in order to calculate MPC input, the following optimal control problem is solved:

\begin{tabular}{cc} 
Problem 1: Model predictive control \\
\hline Minimize & $J_{m p c}$ \\
with respect to & $u$ \\
subject to & Eqs. (21) (26), (30)
\end{tabular}

3.4 Sliding mode control ${ }^{(11)}$

To cope with unknown external disturbances $d_{v}, d_{\beta}$ and $d_{\gamma}$, we apply SMC. Using this control technique, Eqs. (24)(25)(26) are achieved in finite time under the effect of disturbances ${ }^{(12)}$.

Sliding mode functions are defined to express the difference between actual and ideal value as follows:

$$
\begin{gathered}
\sigma_{v}=\frac{1}{\tau s+1}\left(s v-v u_{v}\right), \\
\sigma_{\beta}=\frac{1}{\tau s+1}\left(s \beta-\left(g_{\beta}-\gamma\right)\right), \\
\sigma_{\gamma}=\frac{1}{\tau s+1}\left(s \gamma-g_{\gamma}\right),
\end{gathered}
$$

where $s$ is the Laplace operator and $\tau>0$ is a time constant. It is noted that if $\sigma_{v} \equiv 0, \sigma_{\beta} \equiv 0$ and $\sigma_{\gamma} \equiv 0$ are achieved, Eqs. (24)(25) (26) are realized. To achieve $\sigma_{v} \equiv 0, \sigma_{\beta} \equiv 0$ and $\sigma_{\gamma} \equiv 0$, the inputs of SMC are calculated as follows:

$$
\begin{gathered}
F_{v, s m c}=m \sigma_{v}-\left(\bar{d}_{v}+\epsilon_{v}\right) \operatorname{sgn}\left(\sigma_{v}\right), \\
F_{\beta, s m c}=m v \sigma_{\beta}-\left(\bar{d}_{\beta}+\epsilon_{\beta}\right) \operatorname{sgn}\left(\sigma_{\beta}\right), \\
F_{\gamma, s m c}=I_{z} \sigma_{\gamma}-\left(\bar{d}_{\gamma}+\epsilon_{\gamma}\right) \operatorname{sgn}\left(\sigma_{\gamma}\right),
\end{gathered}
$$

where $\bar{d}_{v}, \bar{d}_{\beta}$ and $\bar{d}_{\gamma}>0$ are upper bounds of disturbance and $\epsilon_{v}, \epsilon_{\beta}$ and $\epsilon_{\gamma}$ are positive constants, which determine speed to achieve $\sigma_{v} \equiv 0, \sigma_{\beta} \equiv 0$ and $\sigma_{\gamma} \equiv 0$. In order to suppress the chattering phenomenon, saturation function is introduced instead of the signum function.

\subsection{Tire force allocation control}

In this section, we present the tire force allocation technique to calculate the tire force $\hat{f}_{x i}$ and $\hat{f}_{y i}$ that achieve the designed generalized forces $F_{v}, F_{\beta}$ and $F_{\gamma}$.

To obtain the generalized forces, there is a redundancy of the tire forces $\hat{f}_{x i}$ and $\hat{f}_{y i}$. Hence we apply an optimization technique considering tire workloads ${ }^{(4)}$. The cost function that expresses the total workload of four wheels is defined as follows

$$
J_{c a}=\sum_{i=1}^{4} \frac{\hat{f}_{x i}^{2}+\hat{f}_{y i}^{2}}{\left(\mu_{i} f_{z i}\right)^{2}} .
$$

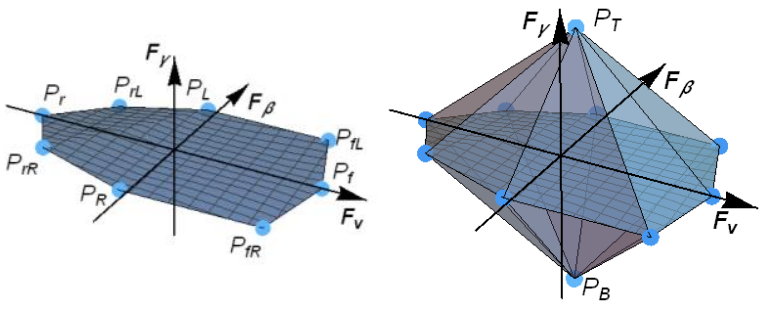

(a) Eight vertices

(b) Hexadecahedron

Fig. 5 Feasible generalized force set $\mathcal{U}$

In order to consider the approximated friction circle $\hat{\mathcal{F}}_{i}$, we introduce constraint for the tire force $\hat{f}_{x i}$ and $\hat{f}_{y i}$ as follows:

$$
\hat{f}_{i}:=\left[\hat{f}_{x i}, \hat{f}_{y i}\right]^{T} \in \hat{\mathcal{F}}_{i} .
$$

In summary the control allocation problem is described as the following constrained quadratic programming $(\mathrm{QP})$ :

$$
\begin{array}{ll}
\frac{\text { Problem 2: Control Allocation }}{\text { Minimize }} & \\
\text { with respect to } & J_{c a} \\
\text { subject to } & \hat{f}_{x i}, \hat{f}_{y i}(i=1,2,3,4) \\
(7)(8)(9)(41)
\end{array}
$$

3.6 Calculating tire command ${ }^{(11)}$

In this section, we present how to calculate slip ratios $\kappa_{i}$ and steering angles $\delta_{i}$, which generate the tire forces $\hat{f}_{x i}$ and $\hat{f}_{y i}$.

To calculate the tire commands $\kappa_{i}$ and $\delta_{i}$, it is required to consider nonlinearity of tire model. Hence we formulate the nonlinear optimization problem and use sequential quadratic programming (SQP) method. The objective function which expresses the error of tire force is set as follows:

$$
\begin{aligned}
J_{w c, i}= & \left(f_{x i} \cos \delta_{i}-f_{y i} \sin \delta_{i}-\hat{f}_{x i}\right)^{2} \\
& +\left(f_{x i} \sin \delta_{i}+f_{y i} \cos \delta_{i}-\hat{f}_{y i}\right)^{2} .
\end{aligned}
$$

In addition constraints are introduced to consider the limitation of actuators. In summary constrained nonlinear problem is described as follows:

\section{Problem 3: Wheel Control

Minimize \\ with respect to \\ $J_{w c, i}$ subject to$$
\left|\kappa_{i}\right| \leq 1,\left|\delta_{i}\right| \leq \bar{\delta}_{i}
$$

\subsection{Traction controller}

In this section, we present the torque controller called traction controller to achieve the slip ratio $\kappa_{i}$ calculated in section 3.6.

A number of traction control techniques are studied ${ }^{(15)}$ to enhance vehicle longitudinal performance. In this paper, to achieve the designed longitudinal force $f_{x i, r}$ calculated based on the slip ratio $\kappa_{i}$ and the steering angle $\delta_{i}$ without steady state error, following traditional PI controller is introduced with feedback gains $K_{p}$ and $K_{i}$ :

$$
\tau_{i}=K_{p}\left(f_{x i}-f_{x i, r}\right)+\frac{K_{i}}{s}\left(f_{x i}-f_{x i, r}\right) .
$$

\subsection{Camber controller}

In this section, we present active camber control law. Although the lateral force is generated by using steering and camber angle, camber angle also generates the load shift. Then the control system of steering and camber angle is separated.

Camber thrust is a force that is generated to tilt direction by camber angle, and value of the force is proportional to camber tilt angle ${ }^{(14)}$. In turning, centrifugal force leads to a load shift and might cause overturn. Hence it is effective that the camber tilts to rotation center. Moreover to prevent a quick change of camber angle, we introduce a low pass filter (LPF). Accordingly camber control law is formulated as follows: 
Table 2 Vehicle specification

\begin{tabular}{lll}
\hline Element & New mobility & Conventional vehicle \\
\hline \hline Weight & $510 \mathrm{~kg}$ & $1300 \mathrm{~kg}$ \\
Wheel base & $2000 \mathrm{~mm}$ & $2600 \mathrm{~mm}$ \\
Width & $1190 \mathrm{~mm}$ & $1760 \mathrm{~mm}$ \\
Height & $1460 \mathrm{~mm}$ & $1515 \mathrm{~mm}$ \\
\hline
\end{tabular}

\begin{tabular}{lll} 
Element & Symbol & Value \\
\hline \hline Horizon number & $\mathrm{T}$ & 10 \\
Discrete interval & $\mathrm{dt}$ & 0.2 \\
Weight & $Q_{s}$ & $\operatorname{diag}\{0.8,35,20,27,7\}$ \\
\multicolumn{1}{c}{ in section 4.3} & $Q_{f}$ & $\operatorname{diag}\{0.1,45,30,30,20\}$ \\
& $R(i)$ & $\operatorname{diag}\{5,5,10\}$ \\
Weight & $Q_{s}$ & $\operatorname{diag}\{0.1,3,10,150,500\}$ \\
$\quad$ in section 4.4 & $Q_{f}$ & $\operatorname{diag}\{5,10,20,200,700\}$ \\
Weight & $R(i)$ & $\operatorname{diag}\{0.1,0.01,0.0001\}$ \\
$\quad$ in section 4.5 & $Q_{f}$ & $\operatorname{diag}\{0.1,0.5,10,1000,100\}$ \\
Weight & $R(i)$ & $\operatorname{diag}\{0.3,50,100,10000,1000\}$ \\
$\quad$ in section 4.6 & $Q_{s}$ & $\operatorname{diag}\{100,300,5\}$ \\
P-gain in section 3.7 & $Q_{f}$ & $\operatorname{diag}\{10,50,1,10,0.3\}$ \\
I-gain in section 3.7 & $K_{p}$ & $\operatorname{diag}\{20,40,40\}$ \\
Time constant & $K_{i}$ & 0.1 \\
in section 3.8 & & 1 \\
\hline
\end{tabular}

\section{Simulation}

\subsection{JSAE-SICE Benchmark Problem}

In the JSAE-SICE benchmark problem No. $3^{(2)}$, a small new generation $\mathrm{EV}$ that can generate independent driving torque, steering angle and camber angle of each wheel, is introduced as the controlled vehicle. In order to simulate behavior of the new generation $\mathrm{EV}$, full vehicle model including dynamics of suspensions, IWMs and actuators is provided on modeling and simulating tool Dymola. In this paper, we conduct a realistic numerical simulation using the full vehicle model and evaluate the vehicle performance in detail.

Since the requirement of the benchmark is to achieve same maneuverability and stability of the small EV as conventional vehicle, two types of vehicle, the specifications of which are described in Tables 2 and 3, are introduced. In addition the compared target controller ${ }^{(16)}$ that consists of driver steering model and velocity PI controller designed by the benchmark committee is also introduced. It is noted that there is no traction control as the velocity PI controller achieves sufficient velocity tracking performance without traction control. In addition, there is also no camber control mechanism in the compared target controller as the conventional vehicle cannot modify the camber angle. The tire parameter used in this benchmark is modified to express the feature of the low RRC tire which tends to have reduced lateral stiffness and maximum tire force ${ }^{(1)}$. In this benchmark problem, to evaluate total maneuverability and stability, four kinds of typical test scenarios are designed: acceleration / deceleration while the cornering, ISO double lane change and run straight while crossing side wind.

\subsection{Simulation settings}

To compare the control performance, we show the three types of simulation results: small EV controlled by proposed method, conventional car controlled by compared target controller and small EV controlled by compared target controller. In the figures these results are drawn with a key "Proposed", "Conv. car" and "Small EV", respectively. In addition to discuss efficient use of tire force, the workload of each tire $\eta_{i}$ is introduced with maximum tire force $\bar{f}_{i}$ as follows:

$$
\eta_{i}=\frac{\left\|\hat{f}_{i}\right\|_{2}}{\bar{f}_{i}} .
$$

$$
Q(i)=\left\{\begin{array}{lc}
Q_{s} & (i=1 \ldots T-1), \\
Q_{f} & (i=T) .
\end{array}\right.
$$

The settings of the MPC parameters, which are empirically designed in order to clearly show the effect of proposed controller, are shown in Table 4. It is noted that the essential effects of proposed method such as considering friction circle and achieving high robustness, would be obtained without fine tuning because friction circle is treated as the constraint in MPC and robustness is mainly due to the effect of SMC. In this paper, it is assumed that all state variables are directly accessible by the controller.

In the full vehicle model simulation tool Dymola, there are many parameters to be initialized. However, it is difficult to compatibly design the initial values of every variable such as suspension spring length and internal stress of the vehicle. Hence, the approximate initial values of every variable are adopted. The results of this, initial behavior of vehicles could be oscillational.

\subsection{Acceleration scenario}

In this test scenario, a vehicle accelerates to $60 \mathrm{~km} / \mathrm{h}(=16.7$ $\mathrm{m} / \mathrm{s}$ ) on a slippery (coefficient of friction $\mu=0.6$ ) curve of $R=50$ $\mathrm{m}$. It should be mentioned that the small vehicle cannot generate enough centripetal force when vehicle runs at target velocity of 60 $\mathrm{km} / \mathrm{h}$ on the curve of $R=50 \mathrm{~m}$. In such a situation, tracking error should be respected, because lane deviation would be cause of an accident in real situation. Hence we suppress the vehicle velocity to reduce the required centripetal force and achieve a high path tracking performance.

The simulation results are depicted in Fig. 6. Although the vehicle cannot run on the target path with target velocity, the high path tracking performance is achieved by using proposed method, which explicitly considers the generalized force limitation, as indicated in Fig. 6 (a). On the other hands, because the vehicle suppresses acceleration to keep the required centripetal force small, the vehicle has stationary velocity tracking error, as indicated in Fig. 6 (b). These results are the contribution of MPC. Fig. 6 (c) (d) show the vehicle side slip angle and yaw rate are suppressed, because the vehicle has small lane deviation and efficiently generate the rotational force. We see the effect of initializing problem from Fig. 6 (f). As indicated in Fig. 6 (g), the camber angle tilt to rotation center, and generate the camber thrust. The vehicle generates the enough centripetal force in the steady 
state. In addition the longitudinal force $F_{v}$ is generated in order to suppress the effect of running resistance at the end of simulation. Fig. 6 (j) shows the work load is about 0.6, because the ratio between maximum longitudinal and lateral force is about 0.6. Then, when vehicle generates the maximum lateral force, the work loads of each wheel are limited about 0.6. After $5 \mathrm{~s}$ the constraint of force limitation Eq. (30) becomes active. These results imply the vehicle cannot generate additional lateral force with the low RRC tire that has oval friction circle depicted in Fig. 3. Thus high cornering and acceleration performance are demonstrated under the friction circle constraint.

\subsection{Deceleration scenario}

In this test scenario, a vehicle decelerates to $0 \mathrm{~km} / \mathrm{h}$ on a slippery split coefficient of friction road $(\mu=0.4$ and 0.9$)$ curve of $R=50 \mathrm{~m}$. It is noted that the small vehicle cannot generate enough centripetal force on the target circular arc with initial velocity of $60 \mathrm{~km} / \mathrm{h}$.

The simulation results are depicted in Fig. 7. In Fig. 7 (c), some lines are interrupted at $5 \mathrm{~s}$, because the side slip angle cannot be calculated when velocity is too small. We see from Fig. 7 (a), the small vehicles ("Proposed" and "Small EV") generate path tracking errors due to centrifugal force. It is because that the maximum lateral force of the small vehicles is smaller than the centripetal force at the initial state. As indicated in Fig. 7 (b), vehicle velocity converges to the target velocity. The vehicle controlled by the proposed method achieves preferred cornering performance such as suppressing the side slip angle and rapid yaw rate change under the small generalized force limitation comparing with the response of "Small EV", as indicated in Figs. 7 (c) (d). The oscillatory torque response in Fig. 7 (h) is caused by the feedback using oscillatory actual tire force in traction control. Some workloads of each tire are smaller than 0.6, as Fig. 7 (j), but it should be emphasized that the constraint of generalized force limitation Eq. (30) kept to active until about $6.5 \mathrm{~s}$. Thus the effectiveness of considering friction circle is shown.

\subsection{Double lane change scenario}

In this test scenario, a vehicle performs ISO double lane change at the target speed of $60 \mathrm{~km} / \mathrm{h}(=16.7 \mathrm{~m} / \mathrm{s})$. In this task, it is necessary to run between the pylons indicating the restriction of the width. Hence we introduce the target path that passes through the pylons combining the straight and quintic functions in order to continuously generate a generalized force. The target path information is considered as the reference state $\xi_{r}$ in MPC.

The simulation results are depicted in Fig. 8. Fig. 8 (a) shows vehicles run between the pylons successfully. As indicated in Fig. 8 (b), although there are modeling errors like running resistance, the target velocity is achieved without steady state error and overshooting. We see from Fig. 8 (c) (d) the small EV controlled by driver model generates the oscillatory rotational behavior after the second lane change (at $9 \mathrm{~s}$ ). On the other hand, superfluous oscillating behavior is suppressed because the new generation EVs can generate the input for vehicle yaw direction using IWMs. In Fig. 8 (c), the side slip angle is large, because the direction of each steering angle is same. Although lateral tire forces are saturated while vehicle changes the lane, the vehicle successfully changes the lane and is not affected by the tire force saturation. From these results, we conclude that the vehicle rotational performance including sideslip and yaw rate response is enhanced using the proposed controller.

\subsection{Side wind scenario}

In this test scenario, a vehicle runs a straight line at target speed of $60 \mathrm{~km} / \mathrm{h}(=16.7 \mathrm{~m} / \mathrm{s})$ while crossing a strong side wind $(20 \mathrm{~m} / \mathrm{s})$. The evaluated point on this scenario is to suppress the effect of disturbance.
The simulation results are depicted in Fig. 9. We see from Fig. 9 (a) the vehicles controlled by driver model are pushed by the side wind and result in the large lateral displacement. In spite of such a hard simulation condition, the resulting deviation is only about $6.2 \mathrm{~mm}$ using the proposed controller. Fig. 9 (b) shows the vehicle velocity converges to the target one, as with the double lane change scenario. As indicated in Fig. 9 (a) (b) (c) (d), effect of wind disturbance is sufficiently negligible. These results are the contribution of SMC which works to suppress the effect of disturbances, as indicated in Fig. 9 (j); during 3 through $6 \mathrm{~s}$ generating the lateral and rotational force to suppress the effect of side wind, and continually generating the longitudinal force to compensate the running resistance. As the contribution of SMC, the magnitudes of sliding mode functions and the effect of disturbance is suppressed sufficiently small, as indicated in Fig. 9 (j). Thus the stability of small EV is increased using the proposed controller.

\section{Conclusion}

In this paper, we show the realistic simulation results that indicate some enhanced tracking performance of the new generation EVs using the proposed controller. In the proposed controller, the tire force limitation is explicitly considered by using the polygonal approximation. Using this approximation, MPC is formulated as a linear optimization (Problem 1); it is expected to reduce the computational cost. In addition the performance of the new generation $\mathrm{EV}$ is brought out with traction and camber controller. The verification is conducted using the full vehicle model with the practical simulation condition given by the vehicle modeling and control committee of the JSAE and the SICE. Through the simulation results, we show the effect of considering friction circle by MPC and the high robustness against the unexpected disturbance by using SMC. This paper shows the first result successfully applied to all simulation scenarios of the JSAE-SICE benchmark problem. Since the purpose of this paper and the JSAE-SICE benchmark problem is to compare the maneuverability and stability of the proposed controller and target performance, the quantitative evaluation about each contribution of adding rotational control input $F_{\gamma}$, prediction of MPC and considering friction circle by MPC is insufficient. Hence, further study is needed to clearly evaluate these contributions in various practical situations. A future direction of this study will be to consider a driver command and to suppress the energy consumption.

\section{References}

(1) I. Kuwayama, H. Matsumoto, H. Heguri : Experimental and Numerical Analysis of the Conceptual Next Generation Ecology Tire (First Report): SAE Int. J. Passeng. Car, Vol. 6, No.2, pp.714-731, (2013)

(2) Y. Hirano : Integrated control of vehicle dynamics and energy consumption - Automotive control and modeling: Benchmark problem No. 3: Proceedings of JSAE Annual Congress Autumn, No. 146-11, pp. 9-12, (2011) (in Japanese).

(3) O. Harkegard, S. T. Glad : Resolving actuator redundancy optimal control vs. control allocation: Automatica, Vol.41, pp.135-144, (2005)

(4) O. Mokhiamar, M. Abe : Simultaneous Optimal Distribution of Lateral and Longitudinal Tire Forces for the Model Following Control: Journal of Dynamic Systems, Measurement, and Control, Vol.126, pp.753-763, (2004).

(5) E. Ono, Y. Hattori, Y. Muragishi, K. Koibuchi : Vehicle Dynamics Integrated Control for Four-wheel-distributed steering and four-wheel distributed traction/braking systems: 


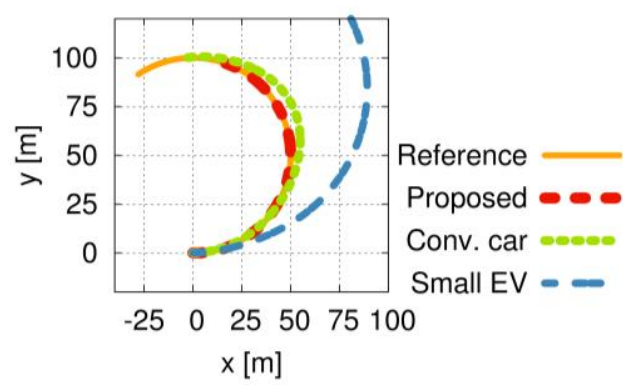

(a) Vehicle trajectory

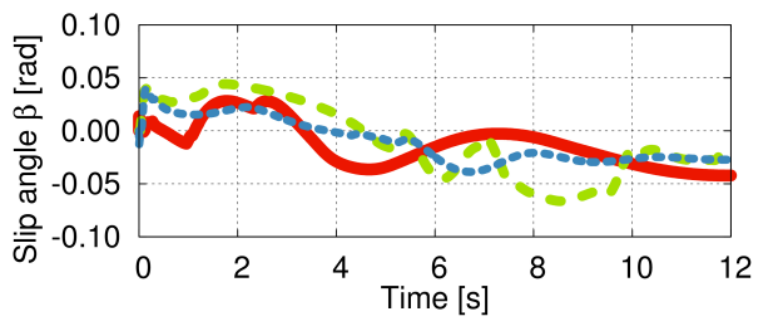

Proposed (c) Vehicle side slip angle

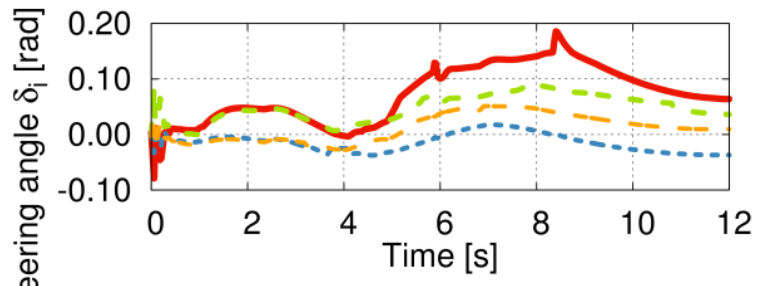

Front Left $-\quad$ Rear Left -
Front Right -
Rear Right - --

(e) Steering angle (Proposed)

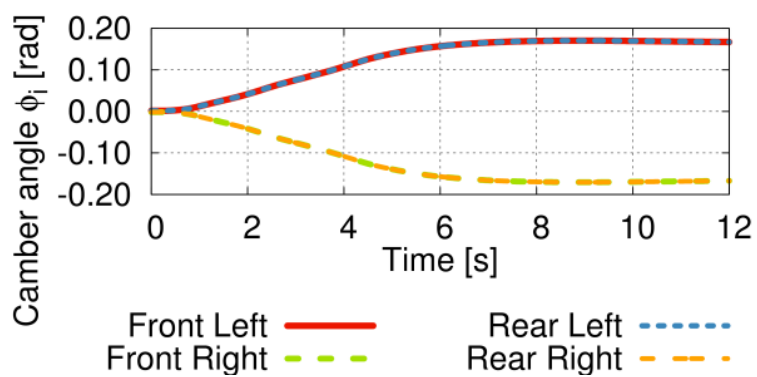

(g) Camber angle (Proposed)

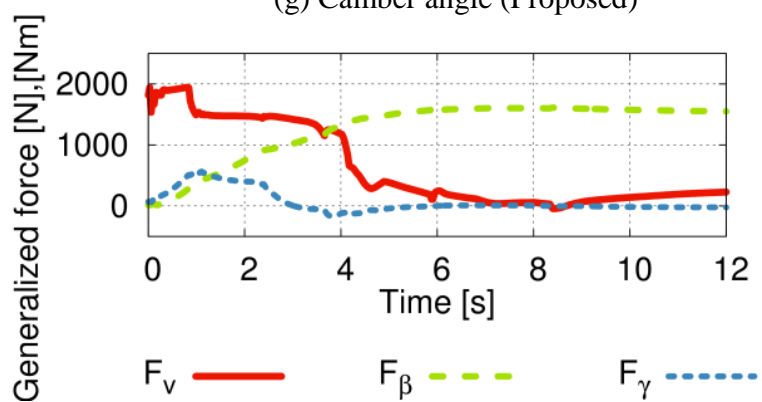

(i) Generalized force (Proposed)

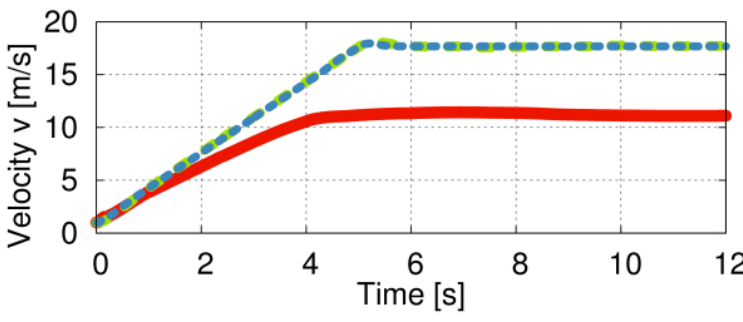

Proposed $\longrightarrow$ Conv. car $-\infty$ Small EV $\cdots \cdots$

(b) Vehicle velocity

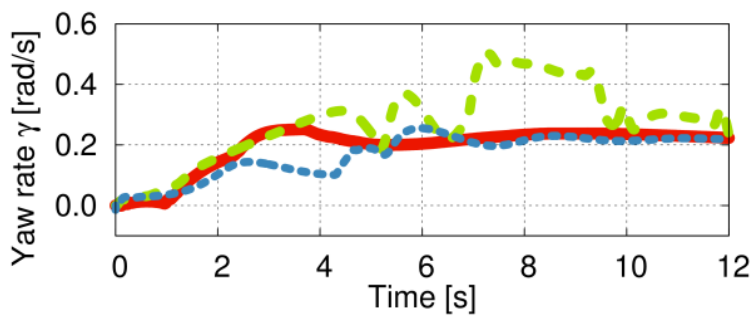

Proposed Conv. car $-\infty-$ Small EV

(d) Vehicle yaw rate

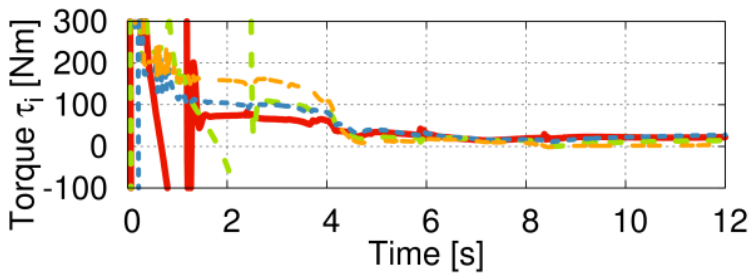

Front Left $\longrightarrow \quad$ Rear Left -.... Front Right - - $\quad$ Rear Right - --

(f) Torque (Proposed)

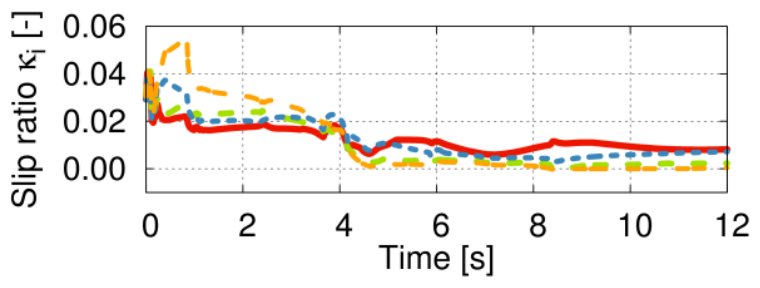

Front Left $\longrightarrow \quad$ Rear Left $\ldots \ldots$ Front Right - - $\quad$ Rear Right - -- -

(h) slip ratio (Proposed)

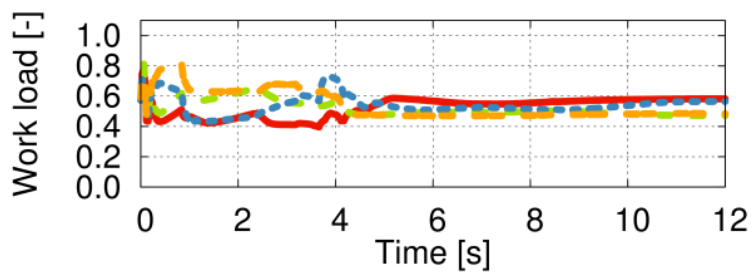

Front-left $\longrightarrow \quad$ Rear-left $\cdots \cdots \infty$ Front-right - - Rear-right - - . (j) Work load (Proposed)

Fig. 6 Acceleration scenario

Vehicle System Dynamics, Vol.44, No.2, pp.139-151, (2006).

(6) P. Falcone, H. E. Tseng, F. Borrelli, J. Asgari, D. Hrovat : MPC-based yaw and lateral stabilisation via active front steering and braking: Vehicle System Dynamics, Vol.46, pp.611-628, (2008).
(7) C. E. Beal, J. C. Gerdes: Model Predictive Control for Vehicle Stabilization at the Limits of Handling: IEEE Trans. On Control Systems Technology, Vol.21, No.4, pp.1258-1269, (2013). 


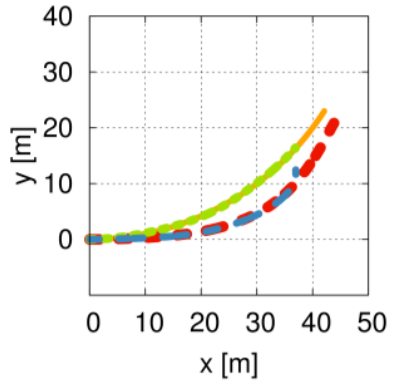

(a) Vehicle trajectory

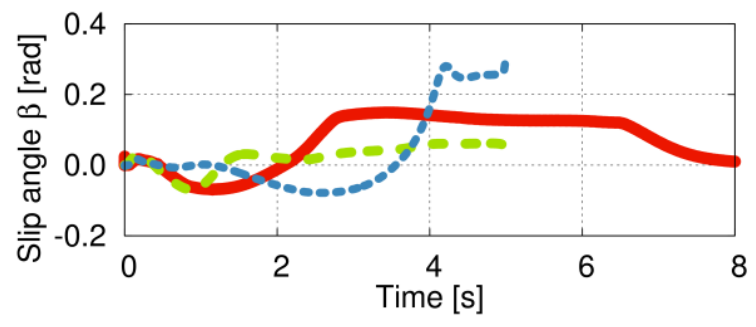

Proposed

(c) Vehicle side slip angle

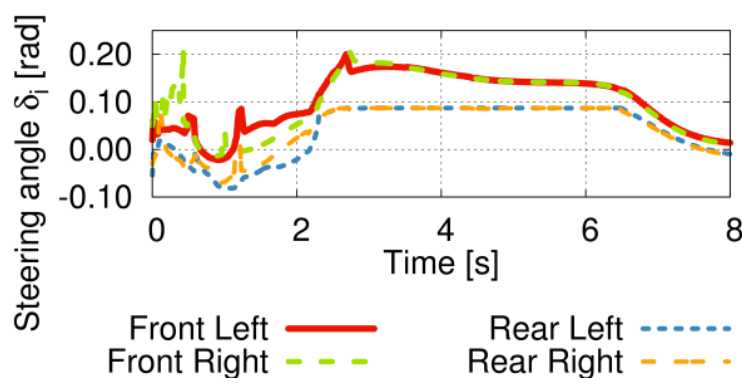

(e) Steering angle (Proposed)

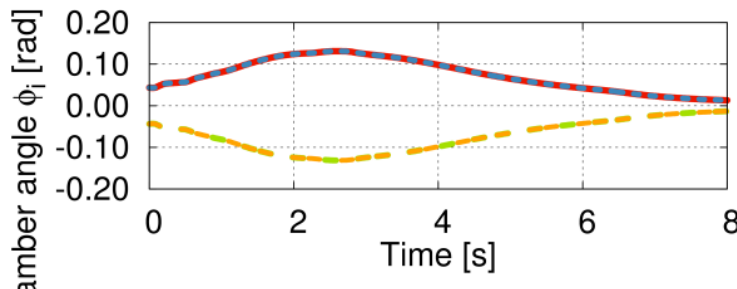

Front Left Rear Left -..-

Front Right - - Rear Right - - -

(g) Camber angle (Proposed)

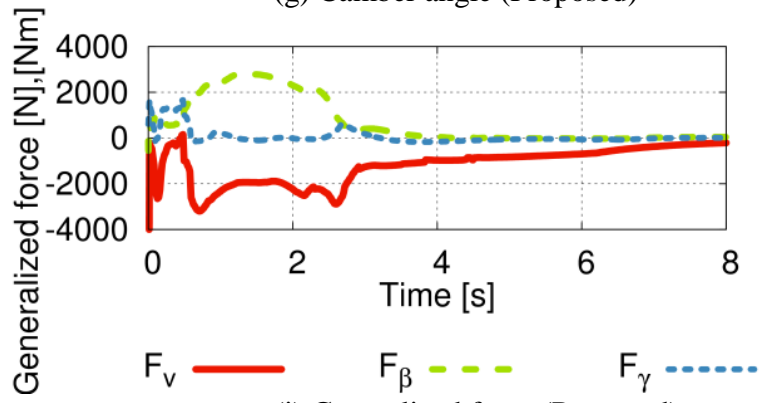

(i) Generalized force (Proposed)

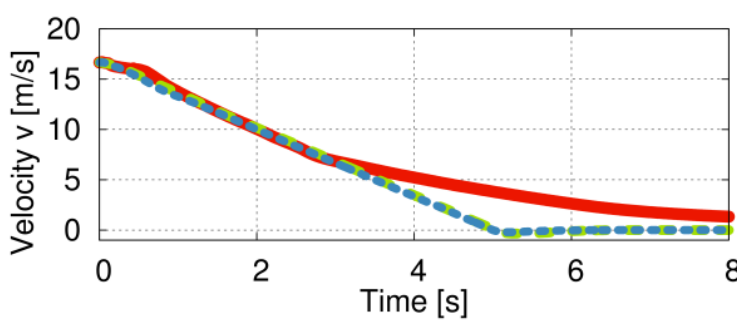

Proposed Conv. car $-\infty$ Small EV

(b) Vehicle velocity

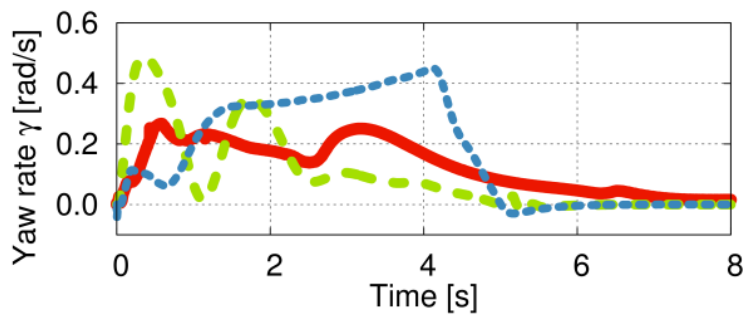

Proposed Conv. car $-\infty$ Small EV - $-\infty$

(d) Vehicle yaw rate

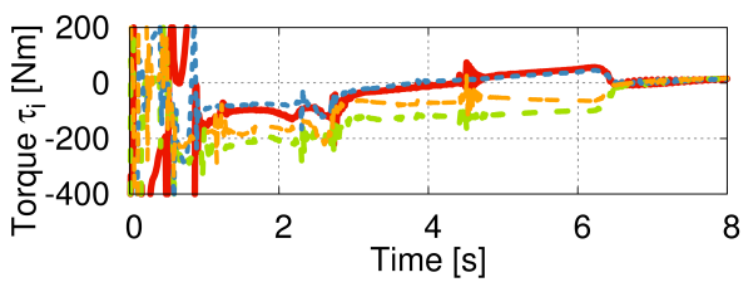

Front Left $-\ldots$ Rear Left $-\cdots$
Front Right $---\quad$ Rear Right - --

(f) Torque (Proposed)

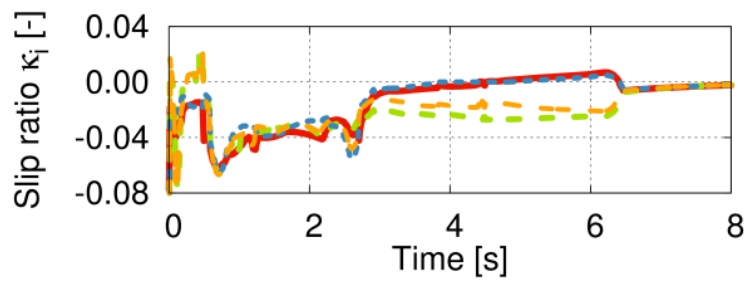

Front Left $\longrightarrow \quad$ Rear Left $\cdots \cdots+$ Front Right - - $\quad$ Rear Right - -- -

(h) slip ratio (Proposed)

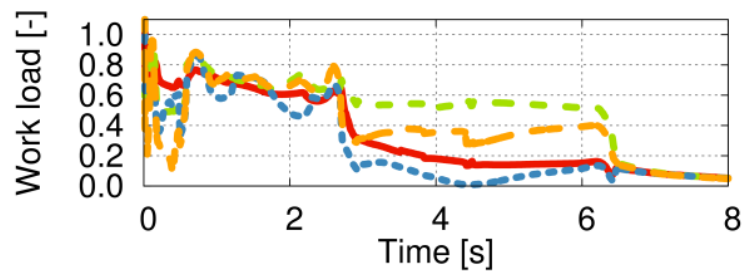

Front-left Rear-left $\cdots \cdots \infty$ Front-right $-\infty \quad$ Rear-right $-\infty$ (j) Work load (Proposed)

Fig. 7 Deceleration scenario

(8) R. Attia, R. Orjuela, M. Basset : Combined longitudinal and lateral control for automated vehicle guidance: Vehicle System Dynamics, Vol.52, No.2, pp.261-279, (2014).

(9) M. Canale, L. Fagiano : Vehicle Yaw Control via SecondOrder Sliding Mode Technique: IEEE Transaction on Industrial Electronics, Vol.55, No.11, pp.3908-3916, (2008).
(10) Z. He, X. Ji : Nonlinear robust control of integrated vehicle dynamics: Vehicle System Dynamics, Vol.49, No.5, pp.657683, (2011). 


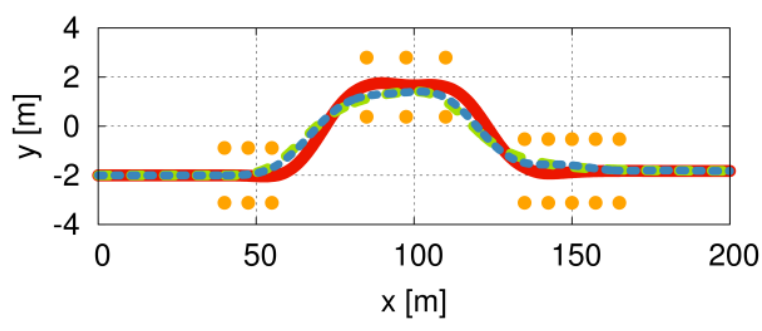

Proposed $\longrightarrow$ Conv. car $-\infty$ Small EV $\cdots \cdots \infty$

(a) Vehicle trajectory

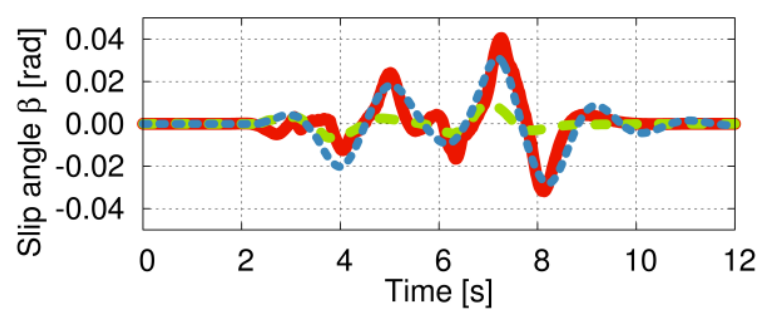

Proposed $\longrightarrow$ Conv. car $-\infty$ Small EV $\cdots \cdots \cdots$

(c) Vehicle side slip angle

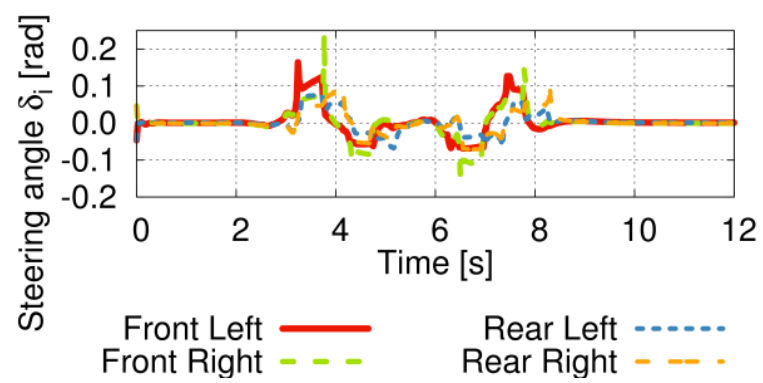

(e) Steering angle (Proposed)

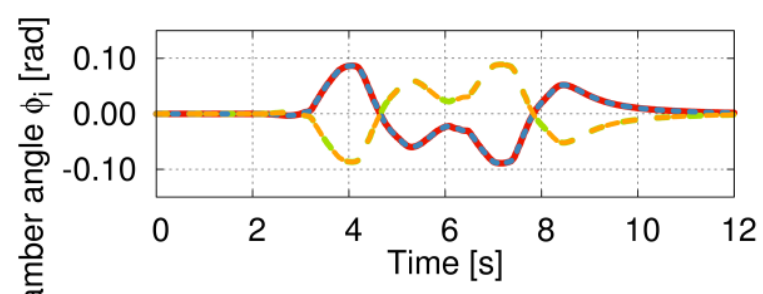

Front Left $-\quad$ Rear Left -.....
Front Right - -

(g) Camber angle (Proposed)

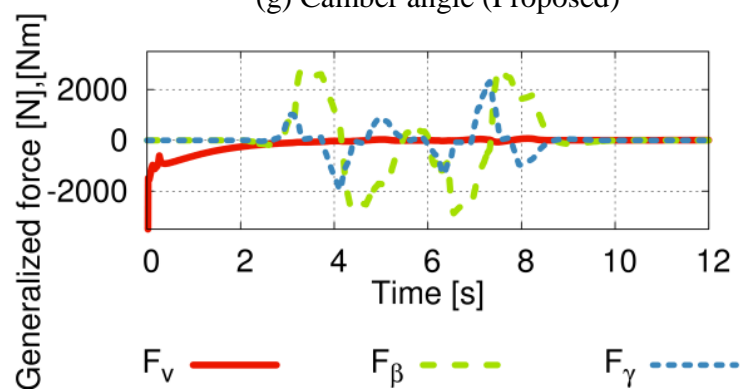

(i) Generalized force (Proposed)

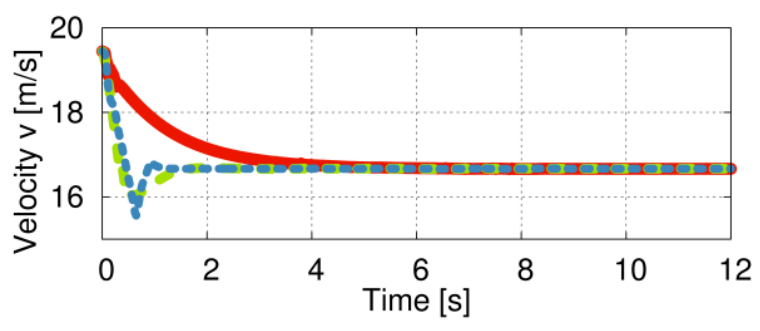

Proposed $\longrightarrow$ Conv. car $-\infty$ Small EV $\cdots \cdots$

(b) Vehicle velocity

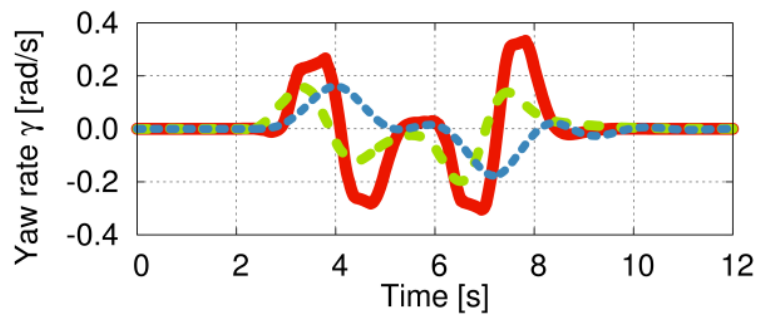

Proposed Conv. car $-\infty$ Small EV $\cdots \cdots$

(d) Vehicle yaw rate

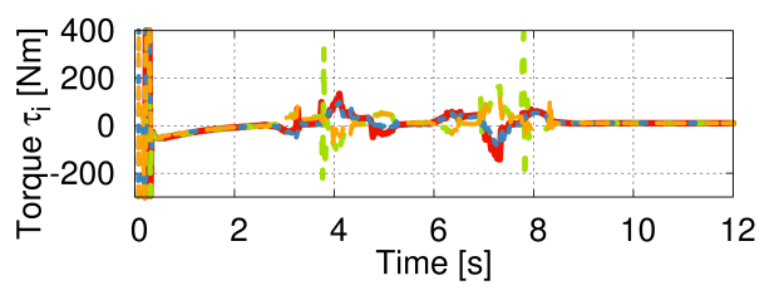

Front Left $\longrightarrow \quad$ Rear Left -..... Front Right - - $\quad$ Rear Right - -- -

(f) Torque (Proposed)

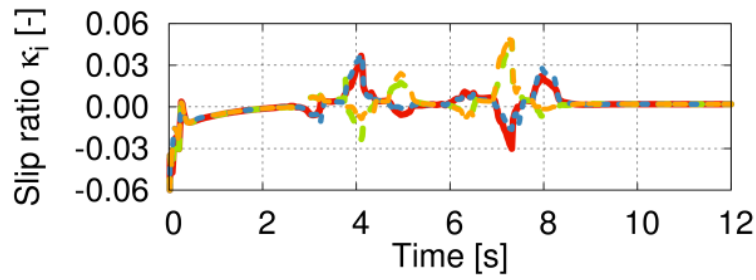

Front Left $\longrightarrow \quad$ Rear Left $\ldots \ldots .$. Front Right - - $\quad$ Rear Right - -- -

(h) slip ratio (Proposed)

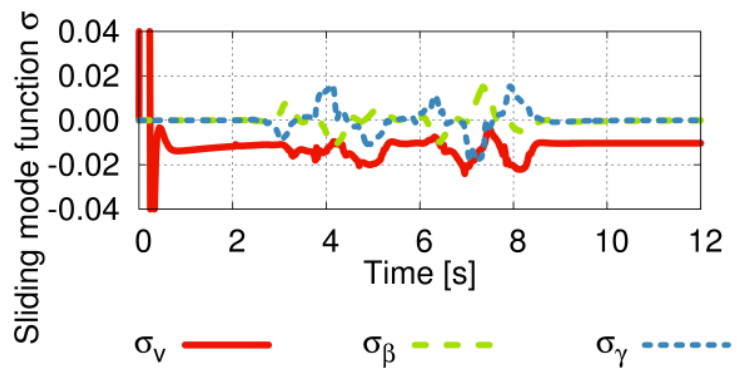

(j) Sliding mode function (Proposed)

Fig. 8 Double lane change scenario

(11) T. Oda, K. Nonaka, K. Sekiguchi: Robust Path tracking Control for Four-wheel Driving and Steering Vehicles using Model Predictive Control and Sliding Mode Control: Trans. of the SICE, Vol.51, No.7, pp.484-493, (2015) (in Japanese).

(12) M. Yamaguchi, T. Asai, Y. Hayakawa, R. Ariizumi : Force Distribution Optimization of Electric Vehicles with
Redundant Degree of Freedom: Proceedings of SICE Annual Congress, pp.6-10, (2016).

(13) S. Yun, H. Nishimura, S. Murakami : Vehicle Dynamics Control System Design by Use of Integrated Control of Front Steering Angle and Driving/Braking Torque for a Micro Four In-Wheel-Motor Vehicle: Trans. of the JSAE, Vol.46, No.2, pp.399-406, (2015) (in Japanese). 


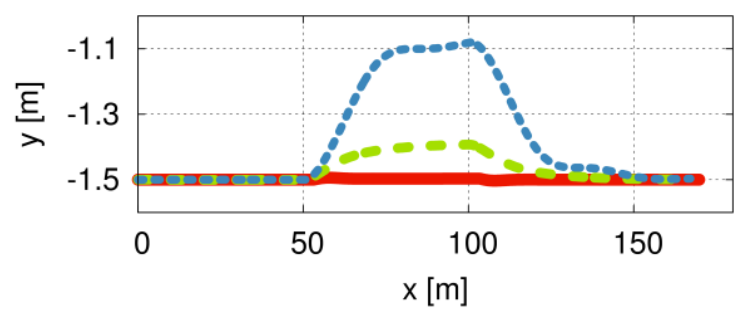

Proposed $\longrightarrow$ Conv. car $-\infty$ Small EV $\cdots \cdots$ (a) Vehicle trajectory

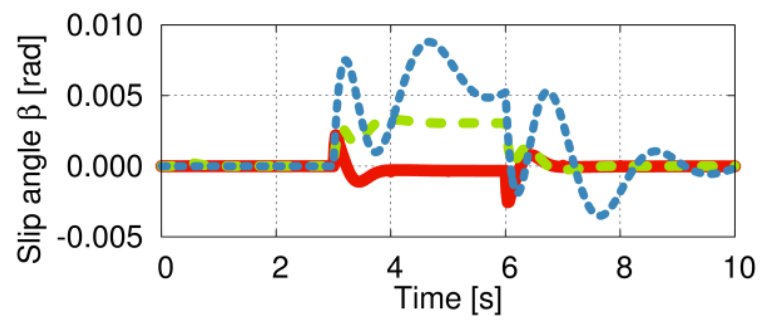

Proposed $\longrightarrow$ Conv. car $-\infty$ Small EV $\cdots \cdots \infty$

(c) Vehicle side slip angle

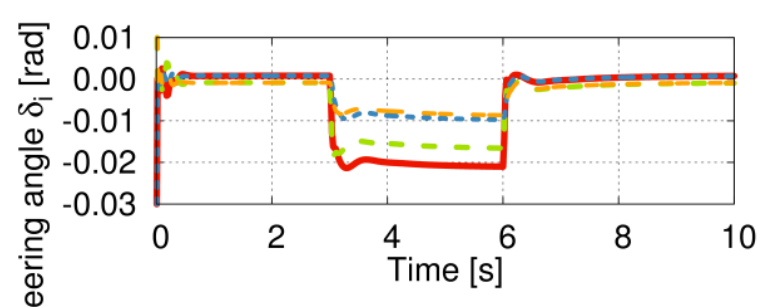

Front Left $---\quad$ Rear Left -
Rear Right - --

(e) Steering angle (Proposed)

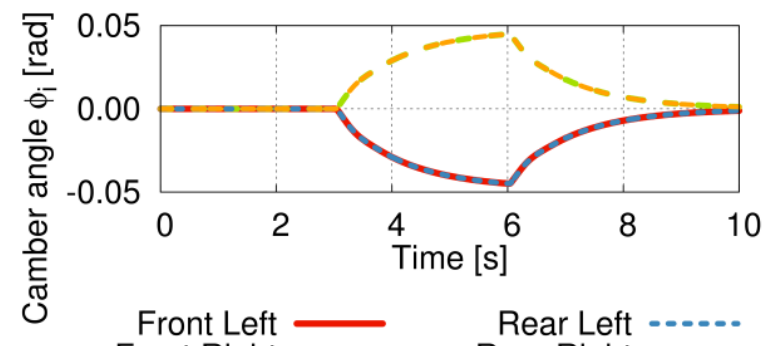

Front Right - - Rear Right - -- -

(g) Camber angle (Proposed)

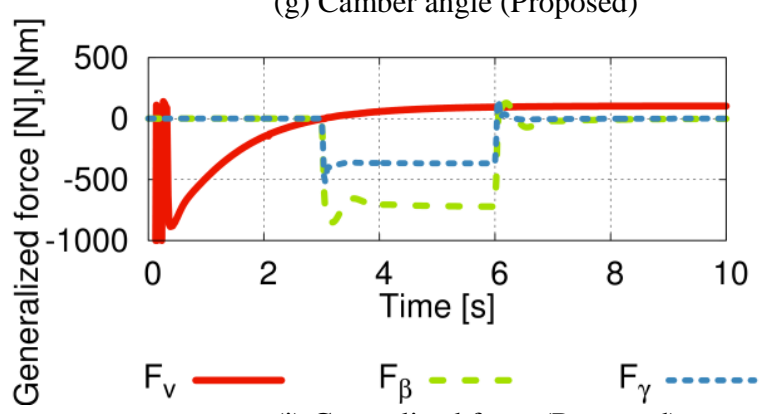

(i) Generalized force (Proposed)

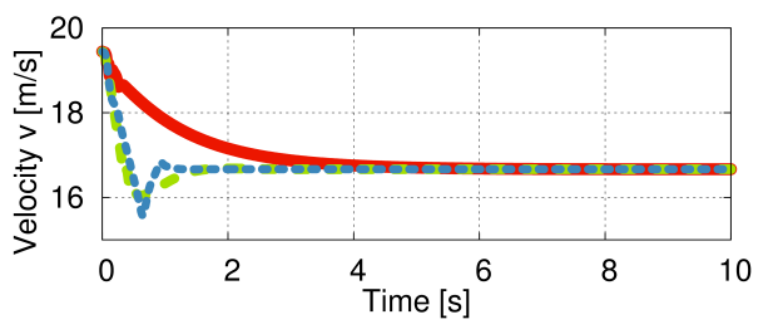

Proposed $\longrightarrow$ Conv. car $-\infty$ Small EV $\cdots \cdots$

(b) Vehicle velocity

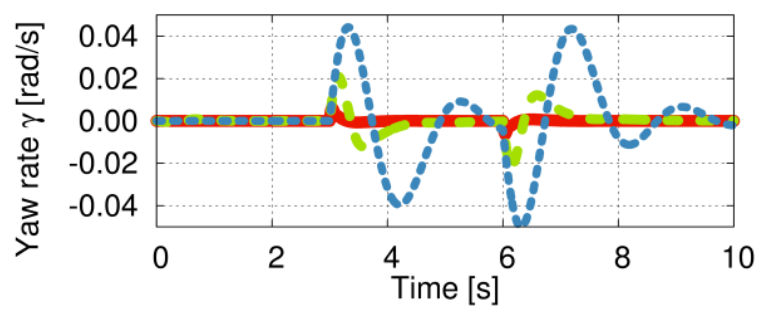

Proposed Conv. car $-\infty$ Small EV $\cdots \cdots$

(d) Vehicle yaw rate

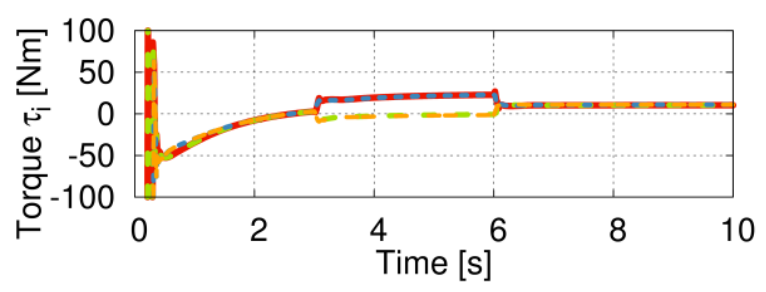

Front Left $-\quad$ Rear Left -
Rear Right - - -

(f) Torque (Proposed)

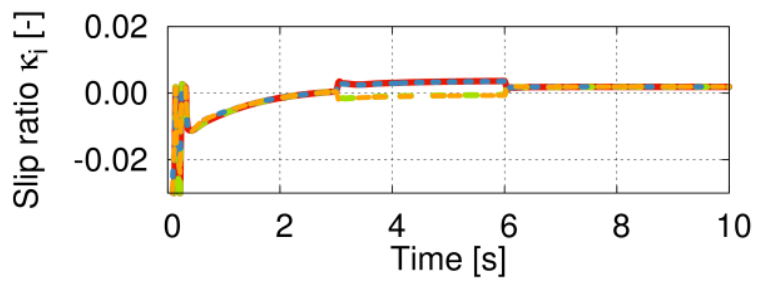

Front Left $\longrightarrow \quad$ Rear Left -..... Front Right - - $\quad$ Rear Right - -- .

(h) slip ratio (Proposed)

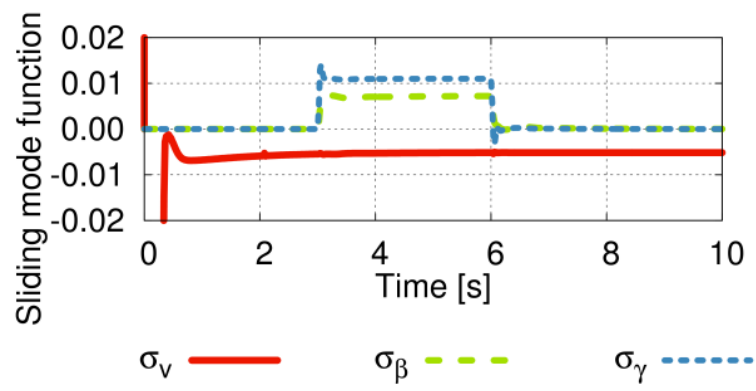

(j) Sliding mode function (Proposed)

Fig. 9 Side wind scenario

(14) H. Pacejka : TIRE AND VEHICLE DYNAMICS: SAE International, (2002).

(15) V. Ivanov, D. Savitski, B. Shyrokau: A Survey of traction control and antilock braking systems of full electric vehicles with individually controlled Electric motors: IEEE Trans. on Vehicular Technology, Vol.64, No.9, pp.3878-3896, (2015).
(16) Y. Hirano : Integrated vehicle control of an in-wheel-motor vehicle to optimize dynamics and energy consumption: World congress on intelligent control and automation, p.2335-2339 (2012) 